\title{
Is there a relationship between periodontal conditions and number of medica- tions among the elderly?
}

\author{
Zuhair S Natto ${ }^{1,2}$, Majdi Aladmawy ${ }^{2}$, Heba K Alshaeri ${ }^{3}$, Mohammed Alasqah ${ }^{4}$ and Athena Papas ${ }^{5}$ \\ ${ }^{1}$ King Abdulaziz University, Faculty of Dentistry, Department of Community Dentistry, Jeddah, Saudi Ara- \\ bia ${ }^{2}$ Tufts University School of Dental Medicine, Department of Periodontology, One Kneeland St, Boston, \\ Massachusetts 02111, United States ${ }^{3}$ Massachusetts College of Pharmacy and Health Sciences, Department of \\ Pharmacology, Boston, Massachusetts, United States ${ }^{4}$ Prince Sattam Bin Abdulaziz University, Department \\ of Preventive Dental Sciences, Alkharj, Saudi Arabia. ${ }^{5}$ Tufts University School of Dental Medicine, Division \\ of Oral Medicine, Boston, Massachusetts, United States
}

DOI: http://dx.doi.org/10.4314/gmj.v50i1.2

Corresponding author: Zuhair S Natto

Conflict of interest: None declared

E-mail: zuhair.natto@tufts.edu

\section{SUMMARY}

Objective: To investigate possible correlations of clinical attachment level and pocket depth with number of medications in elderly individuals.

Methods: Intra-oral examinations for 139 patients visiting Tufts dental clinic were done. Periodontal assessments were performed with a manual UNC-15 periodontal probe to measure probing depth (PD) and clinical attachment level (CAL) at 6 sites. Complete lists of patients' medications were obtained during the examinations. Statistical analysis involved Kruskal-Wallis, chi square and multivariate logistic regression analyses.

Results: Age and health status attained statistical significance $(\mathrm{p}<0.05)$, in contingency table analysis with number of medications. Number of medications had an effect on CAL: increased attachment loss was observed when 4 or more medications were being taken by the patient. Number of medications did not have any effect on periodontal PD. In multivariate logistic regression analysis, 6 or more medications had a higher risk of attachment loss $(>3 \mathrm{~mm})$ when compared to the no-medication group, in crude OR (1.20,95\% CI:0.22-6.64), and age adjusted (OR=1.16, 95\% CI:0.21-6.45), but not with the multivariate model (OR=0.71, 95\% CI:0.11-4.39).

Conclusion: CAL seems to be more sensitive to the number of medications taken, when compared to PD. However, it is not possible to discriminate at exactly what number of drug combinations the breakdown in CAL will happen. We need to do further analysis, including more subjects, to understand the possible synergistic mechanisms for different drug and periodontal responses.

Keywords: periodontal disease, medications, elderly, clinical attachment level, probing depth

\section{INTRODUCTION}

During a human life span, the body will be affected by different kinds of diseases, and with the expanded understanding of disease mechanisms and progression there has been a subsequent expansion in drug treatment. Some of the drugs used will have a direct or indirect impact on the gingiva and periodontal tissue. In the mouth, response to bacterial plaque and activation of the host response is an important defense against microbial pathogens, but this response can be influenced by the medications an individual is taking. ${ }^{1}$

Many studies have investigated the effects of systemic drugs like immunosuppressants ${ }^{2}$ corticosteroids, nonsteroidal antiinflammatory drugs (NSAIDs), ${ }^{3}$ sex hormones $^{4}$ and many others ${ }^{5-7}$ on periodontal health.
Some of these drugs were found to have effects on the periodontal tissue, effects that could decrease ${ }^{8}$ or increase ${ }^{9,10}$ the cellular response of gingival and periodontal tissue to periodontal disease.

Some studies reported that genetic factors were significant in inducing gingival inflammation in association with the use of phenytoin, cyclosporin, and calcium channel blockers. ${ }^{11}$ A systematic review reported on phenytoin and other medications used for epileptic patients, finding associations between phenytoin and gingival hyperplasia (from $16 \%$ to $94 \%$ of cases), and between alveolar bone destruction and phenytoin and carbamazepine. $^{12}$ 
Cyclosporin was tested in animal models to evaluate effects of the drug on periodontal structures; here, histological measurements revealed that soft tissue enlargement and bone desorption were found in rats exposed to cyclosporine. ${ }^{13}$ In a 2013 study, Nassar et al. Induced periodontitis in 3 groups of rats' teeth, finding that a combination of cyclosporine and periodontitis resulted in the most breakdown in the bone height among all 3 groups, and they concluded that cyclosporine interferes and intensifies the imbalance of alveolar bone ridge remodeling in rats. ${ }^{14}$

Following analyses in vitro, other animal studies have reported that corticosteroids induced bone resorption. ${ }^{15}$ A human cross-sectional study evaluating the long-term effects of inhaled corticosteroids reported that corticosteroids might reduce bone remodeling, causing a decrease in bone mineral density. ${ }^{16}$

Another side effect associated with taking multiple drugs is xerostomia, which is associated with inflammation and bleeding from periodontal structures. In a randomized controlled trial, Peker et al (2009) compared healthy patients not taking medication with patients taking medications, and found that the population on medications had statistically significant bleeding and dryness, which Paker et al. associated with the increased amount of missing teeth when compared to the population not taking medications. ${ }^{17,18}$

On the other hand there are drugs that have a favorable biological effect on bone height, such as antibiotics. ${ }^{19}$ Hirsch et al. (2010) presented case reports showing that azithromycin had favorable results in controlling inflammation and regeneration of the alveolar bone. ${ }^{20}$ Metformin is another drug associated with PD reduction and CAL gain when it was used in addition to scaling and root planning. ${ }^{21}$

Thus, previous research indicates that medications have different effects when they are used individually. However, it remains unclear what effects multiple medications, taken together, have on periodontal conditions in the human population, although a few studies have reported on relationships between the number of medications taken and periodontal effects in subjects receiving multiple drug therapy. In India, for example, it was reported that a combination of 2 drugs (neuroleptic + anticholinergic) had the highest association with clinical attachment loss, but increasing numbers of medications were not associated with increased clinical attachment loss. On the other hand, they reported that periodontal probing depth for males increased with the amount of medications taken. ${ }^{22}$
The aim of the current study is to assess for correlations of clinical attachment level and pocket depth with number of medications in an elderly population.

\section{METHODS}

This study recruited volunteers, but the population was not satisfactory. So in order increase minority participation, we recruited additional subjects from the Tufts Geriatric Outreach program (57\% of participants). The Tufts Geriatric Outreach program, conducted at 30 locations in the greater Boston area, includes dental screening, nutritional screening, and educational sessions for elderly people..

Inclusion criteria for enrollment in our study included: being a resident in a community dwelling, retaining six or more teeth, being diagnosed free from terminal illness or endocrine disease affecting nutrition, without recent rapid weight loss, and without active alcoholism. Patients had to be willing and able to complete a 3dimensional food diary in a predetermined manner.

Each participant signed a consent form, agreeing to participate in the study. The study was approved by the Human Investigation Review Committee of Tufts University.

Clinical evaluations were conducted in at Tufts University School of Dental Medicine by an examiner, using artificial light, explorer, mirror, and air syringe. The teeth were free from stains and debris, to allow for better diagnosis.

The coronal and root caries and periodontal measurements were made on all subjects according to the diagnostic criteria used in the US adult survey. Third molars were excluded from examination. Training and calibration sessions to standardize caries and periodontal measurements were held semi-annually. Questionnaires on health knowledge, attitudes and behavior, and general medical and medication history were also administered.

\section{Sample size}

We expected to have $80 \%$ power based on: OR of 2.33 for the no-medication group compared with the medications group, a sample size of 67 , and $\alpha=0.05$. We expected about $50 \%$ drop out due to age of the participant. So, the total sample size was 201 . The sample size was calculated using $G^{*}$ power software, version 3.1 ( University Kiel, Germany). 


\section{Statistical analysis}

Descriptive statistics were expressed as mean $\pm \mathrm{SD}$ or $\mathrm{N}$ $(\%)$. The values of each variable were compared among groups, according to the number of medications. The Shapiro-Wilk test was used to evaluate normality. Kruskal Wallis, chi square and Fisher exact tests were applied.

The odds ratios of increasing number of medication (the main exposure) were used only in the first model. When adjusting for significant variables in other models, the comparison of high clinical attachment level ( $\geq 3 \mathrm{~mm})$ to low $(<3 \mathrm{~mm})$, was estimated using logistic regression. Analyses were performed using SAS 9.3 (SAS Institute, Cary NC).

\section{RESULTS}

We compared demographics and different numbers of medications (Table 1). The demographics of the study population indicated that the mean age for all groups was about 64 years, females, and most of the sample was white, married, and had completed at least 12 years of education. Our subjects had deep clinical attachment level $(\geq 3 \mathrm{~mm})$ and shallow pocket depth $(<3 \mathrm{~mm})$. Age and disease attained statistical significance $(\mathrm{p}<0.05)$ in One Way ANOVA and contingency table analysis (chisquare for independence). Figure 1 shows that we found number of medications to have more effect on clinical attachment level than on pocket depth. We found the same results when we stratified our results by chronic diseases such as cardiovascular disease (CVD), diabetes mellitus (DM), and medically stable (having other diseases or healthy) (Figure 2).

\section{Types of medications}

Cardiovascular medications were the most frequently used medication among this study population (Table 2). Diuretics were the second most frequently used, followed by analgesic and antipsychotic drugs.

\section{Clinical attachment level and number of medications} In a logistic regression model with clinical attachment level ( $\geq 3 \mathrm{~mm}$ vs. $<3 \mathrm{~mm})$ as the outcome variable with number of medications as the main exposure in all models, age only or age with disease status as covariates in the second and third models (Table 3), we found that in the first model clinical attachment levels were more protected between 1 and 4 or 5 medications (all OR < $1.00)$. In other words, taking the required medications will protect the participants from recession to certain extent. However, patients taking 6 or more medications were 1.20 times more likely to have increased recession (OR 1.20, 95\% CI 0.22-6.64).
We found the same pattern after adjusting for age. But when we adjusted for both age and disease status, the pattern disappeared: taking any number of medications was protective against recession.

Table 2 Common medications used among study sample

\begin{tabular}{ll}
\hline Class & Frequency \\
\hline Cardiovascular Agent & 46 \\
Diuretics & 32 \\
Analgesic & 18 \\
Psychotic Drugs & 13 \\
Antibiotic & 13 \\
Antihypertensive & 8 \\
Hypnotic & 4 \\
Respiratory Drugs & 4 \\
Adrenal Glucocorticoid & 4 \\
Antidiabetic & 4 \\
Thyroid Supplement & 4 \\
Female Reproductive Agent & 3 \\
Platelet Aggregation Inhibitor & 3 \\
Antiglaucoma & 3 \\
Antigout & 2 \\
Cholinergic & 2 \\
Anticoagulant & 2 \\
Vitamin Supplement & 2 \\
Gastric Drugs & 2 \\
CNS Agent & 1 \\
Antirheumatic, Cytotoxic & 1 \\
Anti diarrheal & 1 \\
Musculoskeletal Agent & 1 \\
Antivertigo & 1 \\
\hline
\end{tabular}

\section{DISCUSSION}

The population sample size investigated in this report was 139. Almost half of this population was not taking medication $(\mathrm{N}=71)$, while the other half took 1 or more medications $(\mathrm{N}=68)$. Most population individuals were white Caucasians who are married. Other variables, such as CAL, PD, years of smoking, health condition, and years of education, were similar among all the groups.

The relative frequencies of the use of certain medication classes - cardiovascular drugs followed by diuretics, followed by analgesics and anti-psychotics - is not surprising, given the relative frequency of heart and arterial diseases, pain, and mental health issues in elderly populations. $^{23-26}$ 


\section{Original Article}

Table 1 Descriptive statistics of the study sample

\begin{tabular}{|c|c|c|c|c|c|c|c|}
\hline Variable & $\begin{array}{l}\text { No med } \\
\mathrm{N}=71\end{array}$ & $\begin{array}{l}\text { I med } \\
\mathrm{N}=17\end{array}$ & $\begin{array}{l}2 \text { med } \\
N=25\end{array}$ & $\begin{array}{l}3 \text { med } \\
\mathrm{N}=12\end{array}$ & $\begin{array}{l}4 \text { or } 5 \\
N=8\end{array}$ & $\begin{array}{l}6 \text { or more } \\
N=6\end{array}$ & P value \\
\hline Age (mean \pm SD) & $63.68 \pm 10.62$ & $69.0 \pm 5.68$ & $60.52 \pm 12.55$ & $62.67 \pm 11.88$ & $66.63 \pm 9.94$ & $67.86 \pm 12.42$ & $<0.001^{*}$ \\
\hline Gender, $\mathrm{n}(\%)$ & & & & & & & 0.889 \\
\hline Male & $37(52.11)$ & $7(41.18)$ & $11(47.83)$ & $4(36.36)$ & $3(37.50)$ & $3(50.0)$ & \\
\hline Female & $34(47.89)$ & $10(58.82)$ & $12(52.17)$ & $7(63.64)$ & $5(62.50)$ & $3(50.0)$ & \\
\hline Race, $n(\%)$ & & & & & & & 0.436 \\
\hline White & $65(92.86)$ & $16(94.12)$ & $23(92.0)$ & $11(91.67)$ & $7(87.50)$ & $4(66.67)$ & \\
\hline African American & $5(7.14)$ & $1(5.88)$ & $2(8.0)$ & $1(8.33)$ & $1(12.50)$ & $2(33.33)$ & \\
\hline Marital status, $n(\%)$ & & & & & & & 0.415 \\
\hline Single & $9(12.86)$ & $5(29.41)$ & $2(8.7)$ & $3(27.27)$ & $1(12.50)$ & $1(16.67)$ & \\
\hline Married & $40(57.14)$ & $7(41.18)$ & $17(73.91)$ & $7(63.64)$ & $4(50.0)$ & $3(50.0)$ & \\
\hline Others & $21(30.0)$ & $5(29.41)$ & $4(17.39)$ & $1(9.09)$ & $3(37.50)$ & $2(33.33)$ & \\
\hline Clinical attachment level (CAL) (mean \pm SD) & $3.55 \pm 1.08$ & $3.24 \pm 0.95$ & $3.11 \pm 0.73$ & $3.51 \pm 1.37$ & $3.11 \pm 0.49$ & $4.08 \pm 0.49$ & 0.220 \\
\hline Clinical attachment level (CAL) $n(\%)$ & & & & & & & 0.387 \\
\hline$\geq 3 \mathrm{~mm}$ & $23(32.39)$ & $9(52.94)$ & $13(52.0)$ & $5(41.67)$ & $4(50.0)$ & $1(16.67)$ & \\
\hline$<3 \mathrm{~mm}$ & $48(67.61)$ & $8(47.06)$ & $12(48.0)$ & $7(58.33)$ & $4(50.0)$ & $5(83.33)$ & \\
\hline Pocket depth (mean $\pm \mathrm{SD})$ & $2.03 \pm 0.54$ & $2.06 \pm 0.53$ & $2.01 \pm 0.38$ & $2.11 \pm 0.64$ & $2.08 \pm 0.38$ & $2.08 \pm 0.38$ & 0.095 \\
\hline Pocket depth (n,\%) & & & & & & & 0.921 \\
\hline$\geq 3 \mathrm{~mm}$ & $66(92.96)$ & $16(94.12)$ & $24(96.0)$ & 11(91.67) & $8(100.0)$ & $6(100.0)$ & \\
\hline$<3 \mathrm{~mm}$ & $5(7.04)$ & $1(5.88)$ & $1(4.0)$ & $1(8.33)$ & $0(0.0)$ & $0(0.0)$ & \\
\hline Year of smoking (mean $\pm \mathrm{SD})$ & $13.63 \pm 17.37$ & $9.35 \pm 14.12$ & $16.60 \pm 13.72$ & $16.67 \pm 15.98$ & $6.00 \pm 9.30$ & $12.00 \pm 17.32$ & 0.388 \\
\hline Number of cigarettes per day & $7.52 \pm 9.61$ & $6.00 \pm 11.48$ & $16.36 \pm 16.97$ & $9.83 \pm 9.20$ & $5.00 \pm 9.26$ & $7.71 \pm 14.72$ & 0.052 \\
\hline \multirow[t]{2}{*}{ Total number of cigarettes (mean $\pm \mathrm{SD}$ ) } & $79888.73 \pm 120481$ & $43628.24 \pm 77773$ & $133414.80 \pm 19389$ & $75615.83 \pm 80135$ & $34675.00 \pm 68340.9$ & $105745.71 \pm 22731$ & 0.224 \\
\hline & .34 & .50 & 9.91 & 41 & 7 & 6.35 & \\
\hline Disease $(n, \%)$ & & & & & & & $0.030^{*}$ \\
\hline CVD & $12(16.90)$ & $8(47.06)$ & $9(36.0)$ & $2(16.67)$ & $3(37.5)$ & $4(66.67)$ & \\
\hline Diabetes & $16(22.54)$ & $4(23.53)$ & $2(8.0)$ & $3(25.00)$ & $1(12.5)$ & $1(16.67)$ & \\
\hline Medically stable & $43(60.56)$ & $5(29.41)$ & $14(56.0)$ & $7(58.33)$ & $4(50.0)$ & $1(16.67)$ & \\
\hline Year of education $($ mean \pm SD) & $13.30 \pm 2.163$ & $12.59 \pm 2.79$ & $13.30 \pm 2.40$ & $13.90 \pm 2.69$ & $13.63 \pm 3.02$ & $12.17 \pm 4.67$ & 0.884 \\
\hline
\end{tabular}

$*$ p value $<0.05$ 


\section{Original Article}

\begin{tabular}{|c|c|c|c|c|c|c|}
\hline (2) & OR $(95 \%$ CI) & P-value for $\chi^{2 *}$ & OR $(95 \%$ CI $)$ & P-value for $\chi^{2 *}$ & OR $(95 \%$ CI $)$ & P-value for $\chi^{2 *}$ \\
\hline Number of medication & & 0.364 & & 0.354 & & 0.220 \\
\hline No medication & 1 & & 1 & & 1 & \\
\hline 1 & $0.43(0.15-1.25)$ & & $0.41(0.14-1.21)$ & & $0.31(0.10-0.98) \S$ & \\
\hline 2 & $0.44(0.18-1.12)$ & & $0.45(0.18-1.16)$ & & $0.35(0.13-0.93) \S$ & \\
\hline 3 & $0.67(0.19-2.34)$ & & $0.68(0.19-2.36)$ & & $0.67(0.19-2.39)$ & \\
\hline 4 or 5 & $0.48(0.11-2.09)$ & & $0.47(0.11-2.04)$ & & $0.37(0.08-1.73)$ & \\
\hline 6 or more & $1.20(0.22-6.64)$ & & $1.16(0.21-6.45)$ & & $0.71(0.11-4.39)$ & \\
\hline Age & - & & $1.01(0.98-1.04)$ & 0.602 & $1.00(0.97-1.04)$ & 0.880 \\
\hline \multicolumn{7}{|l|}{ Disease } \\
\hline CVD & - & & - & & 1 & \\
\hline Diabetes & - & & - & & $0.30(0.10-0.92) \S$ & \\
\hline Medically stable & - & & - & & $0.39(0.15-1.03)$ & \\
\hline
\end{tabular}

OR, odds ratio; $\mathrm{CI}$, confidence interval.

$* p$ value for trend $\chi^{2}$ test.

$\S p$ value $<0.05$

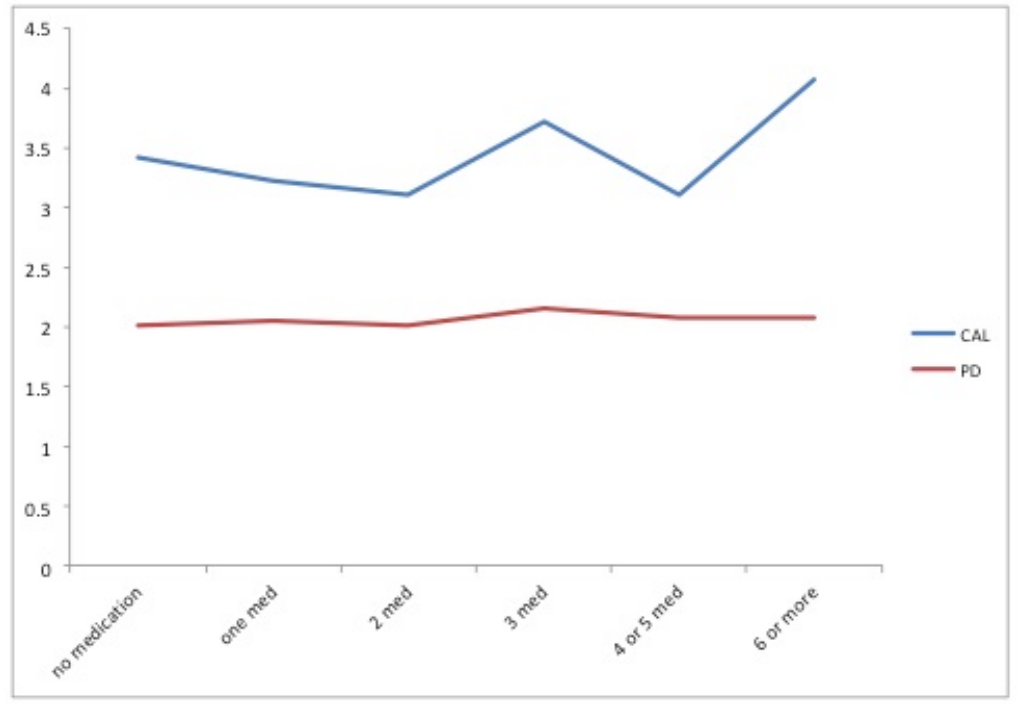

Figure 1 Effect of number of medications on CAL and PD

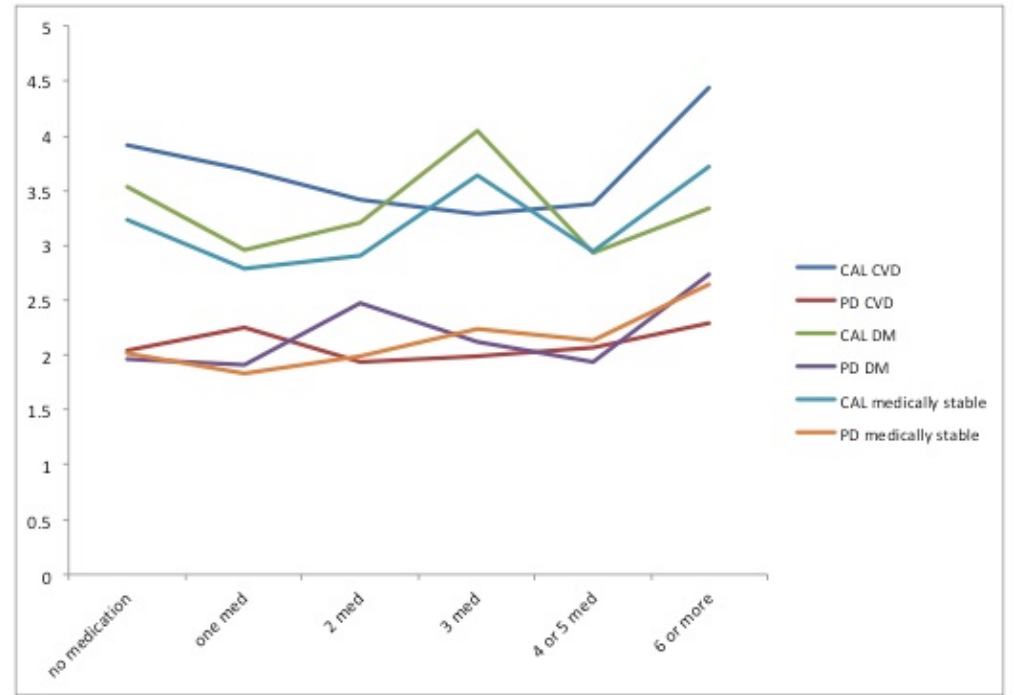

Figure 2 Effect of number of medications on CAL and PD stratified by disease 
The logistic regression analysis used in this study showed that when the number of medications was between 1 and 5 there was a greater likelihood of preventing CAL loss. With $<3 \mathrm{~mm}$ CAL considered healthy or having no previous bone loss, and $>3 \mathrm{~mm}$ considered diseased or having previous bone loss, a change from CAL maintenance to CAL loss was seen in patients taking more than 5 medications; for those patients, there was 1.2 times the chance to develop CAL loss.

On the other hand, periodontal probing depth did not change for any amount of medications taken. This is potentially an important finding, given that between 1998 and 2008 the percentage of patients taking medication who were taking 5 or more drugs increased from $6 \%$ to $11 \%$ in the United States. ${ }^{27}$

In our second model, introducing age as a variable in analysis, the odds ratio for CAL loss $(>3 \mathrm{~mm})$ is 1.16 for patients taking more than 5 medications. Thus, age had no impact in these subjects. When the disease status was included into the model along with the age, none of the medications amount groups had CAL loss. Our results seem to support Ciancio et al's findings (1997, 2005) that while most medications increased the risk of developing caries and periodontal disease, a few medications might decrease the risk of periodontal breakdown. $^{28,29}$

Though our study findings are significant, our research had limitations in terms of sample size and population homogeneity, and further research in multicenter settings is needed to study the relationship between number of medications and CAL.

\section{Conclusion}

This study has demonstrated that multiple-medication regimens in elderly patients are more likely to affect clinical attachment level than pocket depth. It is, however, not possible to discriminate exactly at what drug combination threshold the breakdown in clinical attachment level occurs - this seems dependent upon drug type - but it is likely that intake of 5 or more medications should raise concerns about attachment level. A larger study is needed to investigate this issue further.

\section{ACKNOWLEDGEMENT}

We would like to acknowledge all the patients that were recruited for the study at Tufts University School of Dental Medicine.

\section{REFERENCES}

1. Ebersole JL. Immune responses in periodontal diseases. In: Wilson TG Jr, Kornman KS, editors. Fundamentals of periodontics, 2nd edn. Chicago, IL: Quintessence Publishing Co., Inc, 2003: 111143.

2. Kobayashi T, Okada M, Ito S, Kobayashi D, Ishida K, Kojima A, Narita I, Murasawa A, Yoshie H. Assessment of interleukin-6 receptor inhibition therapy on periodontal condition in patients with rheumatoid arthritis and chronic periodontitis. J Periodontol. $2014 ; 85(1): 57-67$.

3. Alani A, Seymour R. Systemic medication and the inflammatory cascade. Periodontol 2000. 2014 ;64(1):198-210.

4. Shiau HJ, Aichelmann-Reidy ME, Reynolds MA. Influence of sex steroids on inflammation and bone metabolism. Periodontol 2000. 2014 ;64(1):81-94.

5. Meechan JG, Seymour RA. Drug Dictionary for Dentistry 2002. Oxford: Oxford University Press, 2002.

6. Davies DM, Ferner RE. Davies's Textbook of Adverse Drug Reactions, 5th edn. London: Chapman \& Hall Medical, 1998.

7. Royzman D, Recio L, Fiorellini J, Goodson M, Howell H. The effect of aspirin intake on bleeding on probing in patients with gingivitis. J Periodontol 2004: 75: 679-684.

8. Cengiz MI, Sümer P, Cengiz S, Yavuz U. The effect of the duration of the dialysis in hemodialysis patients on dental and periodontal findings. Oral Dis. 2009 ;15(5):336-41.

9. Schuller PD, Freedman HL, Lewis DW. Periodontal status of renal transplant patients receiving immunosuppressive therapy. J Periodontol. 1973: 44: $167-170$.

10. Seymour RA, Ellis JS, Thomason JM. Risk factors for drug-induced gingival overgrowth. J Clin Periodonto.l 2000: 27: 217-223.

11. Livada R, Shiloah J. Calcium channel blockerinduced gingival enlargement. J Hum Hypertens. $2014 ; 28(1): 10-4$.

12. Seymour RA, Thomason JM, Ellis JS. The pathogenesis of drug-induced gingival overgrowth. $J$ Clin Periodontol. 1996 ;23(3 Pt 1):165-75.

13. Cornacchio AL, Burneo JG, Aragon CE. The effects of antiepileptic drugs on oral health. J Can Dent Assoc. 2011;77:b140

14. Nassar PO, Felipetti FA, Nassar CA, Spolidorio LC. Evaluation of effect of cyclosporine A on the bone tissue with induced periodontal disease to ligature in rats. Transplant Proc. 2013 ;45(2):778-82.

15. Bouvard B, Gallois Y, Legrand E, Audran M, Chappard D. Glucocorticoids reduce alveolar and 
trabecular bone in mice. Joint Bone Spine. 2013 ;80(1):77-81.

16. Komerik N, Akkaya A, Yildiz M, Buyukkaplan US, Kuru L. Oral health in patients on inhaled corticosteroid treatment. Oral Dis. 2005 ;11(5):303-8.

17. Peker I, Alkurt MT, Usalan G. Clinical evaluation of medications on oral and dental health. Int Dent $J$. $2008 ; 58(4): 218-22$.

18. Shinkai RS, Hatch JP, Schmidt CB, Sartori EA. Exposure to the oral side effects of medication in a community-based sample. Spec Care Dentist. 2006 ;26(3):116-20.

19. Nibali, L., Pometti, D., Tu, Y.-K. and Donos, N. Clinical and radiographic outcomes following nonsurgical therapy of periodontal infrabony defects: a retrospective study. Journal of Clinical Periodontology, 2011: 38: 50-57.

20. Hirsch R. Periodontal healing and bone regeneration in response to azithromycin. Aust Dent J. 2010 Jun;55(2):193-9.

21. Pradeep AR, Rao NS, Naik SB, Kumari M. Efficacy of varying concentrations of subgingivally delivered metformin in the treatment of chronic periodontitis: a randomized controlled clinical trial. $J$ Periodontol. 2013;84(2):212-20.

22. Gopalakrishnapillai, A. C., Iyer, R. R. and Kalantharakath, T. Prevalence of periodontal disease among inpatients in a psychiatric hospital in India. Special Care in Dentistry. 2012: 32: 196-204.
23. Zdanowicz N, Lepiece B, Tordeurs D, Jacques D, Reynaert C. Predictability of levels of physical and mental health in adults and adolescents: a 2 years longitudinal study. Psychiatr Danub. $2012 ; 24$ Suppl 1:S9-13.

24. Balaoing LR, Post AD, Liu H, Minn KT, GrandeAllen KJ. Age-Related Changes in Aortic Valve Hemostatic Protein Regulation. Arterioscler Thromb Vasc Biol. 2013: 31.

25. Fowkes FG, Rudan D, Rudan I, Aboyans V, Denenberg JO, McDermott MM, Norman PE, Sampson UK, Williams LJ, Mensah GA, Criqui $\mathrm{MH}$. Comparison of global estimates of prevalence and risk factors for peripheral artery disease in 2000 and 2010: a systematic review and analysis. Lancet. 2013: 19;382(9901):1329-40.

26. Natto ZS, Aladmawy M, Alasqah M, Papas A. Factors contributing to tooth loss among the elderly: a cross-sectional study. Singapore Dent $J$. 2014;35(1): 17-22.

27. Gu Q, Dillon CF, Burt VL. Prescription drug use continues to increase: U.S. prescription drug data for 2007-2008. NCHS data brief, no 42. Hyattsville, MD: National Center for Health Statistics. 2010.

28. Ciancio SG. Medications as a risk factor for caries and periodontal disease. NY State Dent J. 1997 ;63(8):32-6.

29. Ciancio SG. Medications: a risk factor for periodontal disease diagnosis and treatment. $J$ Periodontol. $2005 ; 76(11$ Suppl):2061-5 